\title{
Enhancement of Hidrogen Physisorption Energy by Lithium-Doped Carbon Nanotube: A First Principle Study
}

\author{
Ir. Nasruddin, Engkos Achmad Kosasih, Supriyadi, and Ihsan Ahmad Zulkarnain
}

\begin{abstract}
Hydrogen fuel-cell is potential future energy carrier to be implemented on vehicle. However, one of the main problems of its implementation is about how to store Hydrogen in high density at ambient temperature and acceptable pressure. One of the promising storage methods is adsorption on Carbon nanostructured material, one of them is Carbon Nanotube (CNT). However, CNT is still too weak to adsorb Hydrogen gas in ambient temperature. Therefore, many researches have been conducted to find ways to improve Hydrogen physisorption energy on CNT. One of the ways is by doping Lithium cation into CNT. Lithium-cation is expected able to effect electrochemical properties of CNT so that Hydrogen physisorption energy is enhanced. In this study, we performed ab-initio calculations to investigate effect of Lithium-doping on CNT to its Hydrogen physisorption energy. Our research result shows that Lithium-doped CNT have stronger physisorption energy, up to $-4.2 \mathrm{kcal} / \mathrm{mol}$, compared to undoped CNT, only $-1.2 \mathrm{kcal} / \mathrm{mol}$.
\end{abstract}

Index Terms-Hydrogen storage, CNT, Lithium, Ab initio.

\section{INTRODUCTION}

United States Department of Energy (DoE) launch a target for hydrogen storage capacity to be about $5.5 \mathrm{wt} \%$ in ambient temperature and reasonable pressure to make it applicable for fuel-cell powered vehicle [1]. Storage of hydrogen is attempted in various approaches involving gas phase storage with compressed hydrogen gas tanks, liquid hydrogen tanks and in solid state materials like metal hydrides, carbon-based materials/high surface area sorbents such as metal-organic frameworks (MOFs) and chemical hydrogen storage using complex hydrides. Among the various options of hydrogen storage, only storage in solid state materials seems to be promising [2]. Carbon Nanotube (CNT) is the most popular nanostructured material which is potential to be hydrogen storage medium due to its high specific surface area [3]. However, its hydrogen sorption capacity still has not reached DoE Target yet. So, many researches are working on designing modification of any configurations of CNT to enhance its hydrogen storage capacity. Ralph T. Yang reported that Alkali doping, such as Lithium and Kalium, on CNT is able to enhance the hydrogen storage capacity up to 2 $\mathrm{wt} \%$ at room temperature. Lithium doping is able to transfer charge to CNT, therefore its polarizability is increased and affect its adhesive energy to adsorb hydrogen molecules [4]. So, it is necessary to perform further investigation about the effect of Lithium doping on CNT to its hydrogen

Manuscript received November 25, 2013; revised March 26, 2014.

Ir. Nasruddin is with the Department of Mechanical Engineering Faculty of Engineering University of Indonesia (e-mail: ihsan.a.zulkarnain@gmail.com). physisorption characteristics. Due to these backgrounds, we are planning to conduct research on the effect of Lithium doping on hydrogen sorption capacity of Carbon Nanotube through quantum mechanics study.

\section{COMPUTATIONAL DetaILS}

Unlike previous studies by Cho et al. [5] and Han et al. [6] which used DFT-GGA-PW91 method, we use ab-initio method that involve dispersion-term because Hydrogen physisorption on CNT phenomena is dominated by dispersion interaction due to non-polar properties of Hydrogen gas. There are so many options of ab-initio method that involve dispersion-term, such as Moller-Plesset Perturbation Theory (MP2), dispersion-corrected Density Functional Theory (d-DFT), and many other Post Hartree-Fock Methods. In this study, we use MP2 to calculate physisorption energy of Hydrogen on CNT. Basis-sets used in this study is def2-SVP of TURBOMOLE package, equal with $6-31 \mathrm{G}$ polarized and diffused basis-sets of GAUSSIAN package. These double zeta basis-sets are commonly used for explorative study of new material properties. All of the calculations are performed using PSI4 package from sandia Laboratory [7].

$\mathrm{Ab}$-initio calculations are initiated by defining structure of the adsorbent. The adsorbent structures, in this case, CNT, are generated using VMD package and then optimized using AVOGADRO package with Universal Force-field. The adsorbent structure then simplified into smaller system in order to reduce its computational cost for ab-initio calculations. In this study, we investigate Hydrogen physisorption energy in many different carbon-based adsorbent structure, such as Benzene, CNT, and Lithium-doped CNT.

\section{RESUlTS AND DisCUSSIONS}

Before we start investigating Hydrogen physisorption energy in many different adsorbent structures, we also investigate Hydrogen gas intermolecular potential energy to be stated as minimum adsorption energy criteria of Hydrogen gas. This is because adsorption phenomena will occur only when interaction force between adsorbate and adsorbent is stronger than adsorbate-adsorbate interaction. In more simple word, adhesive force must be stronger than cohesive force for adsorption to occur.

\section{A. Interaction Energy of Hydrogen Gas Molecules}

Hydrogen gas molecules are non-polar. But actually, they are have quadrupole moment, where the nucleat of Hydrogen atom is more positive-charged compared to electron-r ‘ich 
region in the bond between Hydrogen atoms. This properties of Hydrogen gas molecules make them may interact with many orientations (see Fig. 1). Our ab-initio calculation results show that Hydrogen gas molecules interacts most stable in T-orientation with interaction potential energy up to $-0.075 \mathrm{kcal} / \mathrm{mol}$ (see Fig. 2).

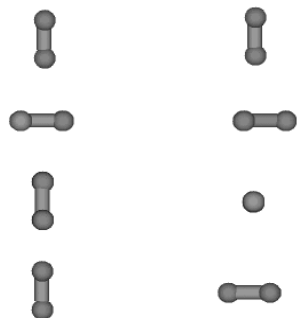

Fig. 1. Orientations of hydrogen gas molecules interaction.

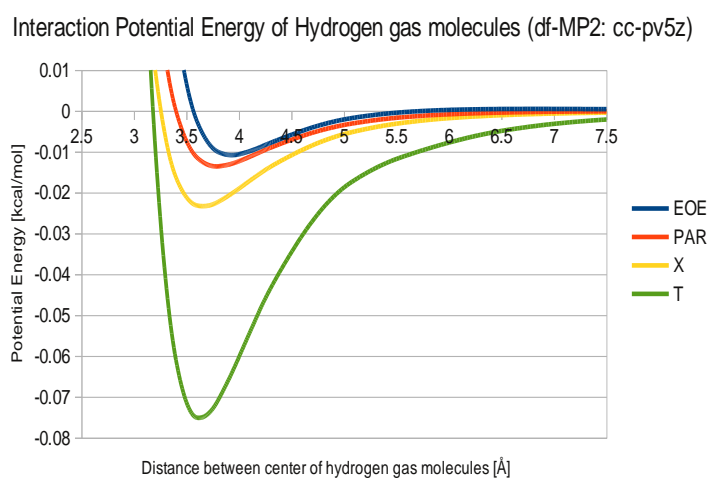

Fig. 2. Interaction potential energy of Hydrogen gas molecules.

\section{B. Hydrogen Physisorption Energy on Benzene}

Before investigating physisorption energy of Hydrogen on larger adsorbent structure, investigating on smaller adsorbent structure, such as Benzene, may be a good choice. The physisorption of Hydrogen on Benzene are performed in two different orientations, parallel and perpendicular (see Fig. 3). The calculations results also show that the perpendicular orientation is more stable $(-1.2 \mathrm{kcal} / \mathrm{mol})$ compared to the parallel orientation $(-0.8 \mathrm{kcal} / \mathrm{mol})$ (See Fig. 4). These results are quite agreeable with others similar works by Zhou et al. [8] $(1.12 \mathrm{kcal} / \mathrm{mol})$

An existence of electron-rich pi-region on Benzene surface makes the more positive-quadrupole-charged Hydrogen atom becomes attracted and the more negative-quadrupole-charged Hydrogen bond becomes repulsed. It is the reason why perpendicular orientation be the most stable state of Hydrogen gas - Benzene interaction.

\section{Hydrogen Physisorption Energy on CNT}

Just like the previous calculations on Benzene, the Hydrogen physisorption energy on SWCNT $(6,0)$ are analyzed by performing ab-initio calculation in two orientations: parallel and perpendicular (see Fig. 5). The calculations results show that Hydrogen physisorption energy on SWCNT is stronger compared to the energy on Benzene (see Fig. 6). It is caused by the existence of much more electron-rich pi-orbital that occur in the system which attract the Hydrogen molecule. The calculated Hydrogen physisorption energy on the CNT is about $-1.2 \mathrm{kcal} / \mathrm{mol}$ in perpendicular orientation and $-0.7 \mathrm{kcal} / \mathrm{mol}$ in parallel orientation.
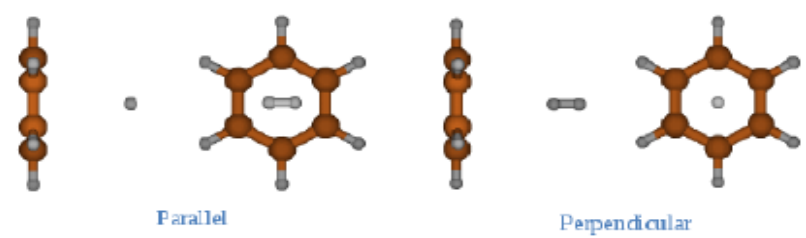

Fig. 3. Orientations of Hydrogen-Benzene interaction.

Interaction Potential of Hydrogen gas - Benzene (df-MP2: def2-SVP)

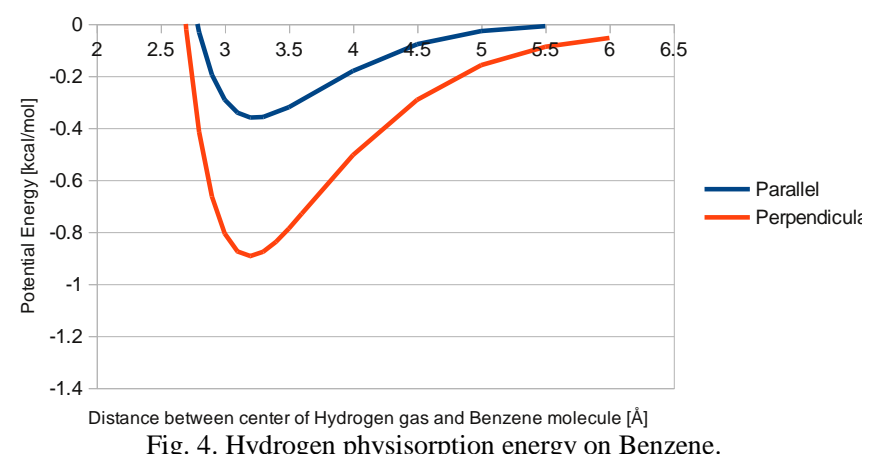

Fig. 4. Hydrogen physisorption energy on Benzene.
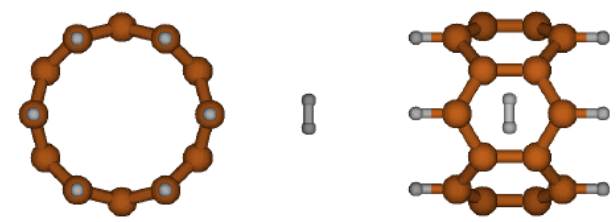

\&
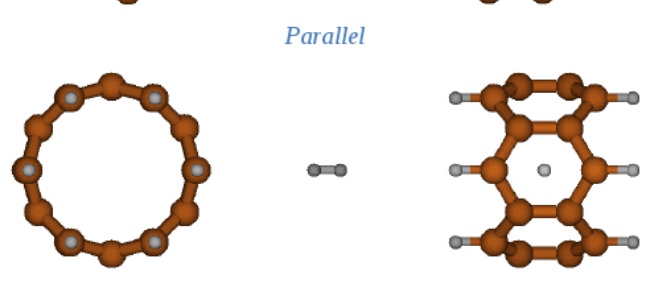

Perpendicular

Fig. 5. Orientations of hydrogen-CNT interaction

Interaction Potential of Hydrogen gas - SWCNT (df-MP2: def2-SVP)

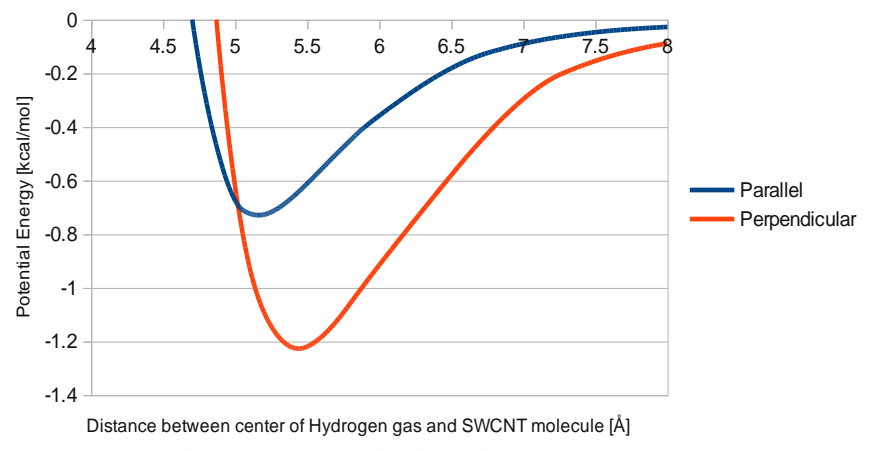

Fig. 6. Hydrogen physisorption energy on CNT.

\section{Hydrogen Physisorption Energy on Lithium-Doped CNT}

Electron-rich pi region on CNT has ability to adsorb cation, such as Lithium. The existence of Lithium-cation on CNT is expected to be able to effect Hydrogen physisorption energy on CNT. Therefore, we investigate it by performing ab-initio calculations for Hydrogen physisorption energy on Lithium-doped CNT in two orientation, parallel and perpendicular. The Hydrogen is positioned on two sites, near Lithium and far from Lithium (see Fig. 7 and Fig. 9). The calculation results show that perpendicular orientation is not the most stable state anymore like some previous calculations 
(see Fig. 8 and Fig. 10). The parallel orientations become the most stable state for Hydrogen physisorption on Lithium-doped CNT with enhancement of physisorption energy up to $-4.2 \mathrm{kcal} / \mathrm{mol}$. This phenomena occur because of the existence of positive-charged Lithium-cation. The electron density of Hydrogen gas molecules that is concentrated on the center of the bond is attracted to Lithium-cation. This interaction is stronger compared to interaction between positively-charged Hydrogen nucleat with electron-rich pi region of CNT, consequently the physisorption energy of Hydrogen is enhanced.

These result bring to a conclusion that Lithium-doping into CNT is theoretically able to enhance Hydrogen physisorption energy so that Hydrogen physisorption storage capacity can be increased too as stated experimentally by Chen and Yang [9], [10].
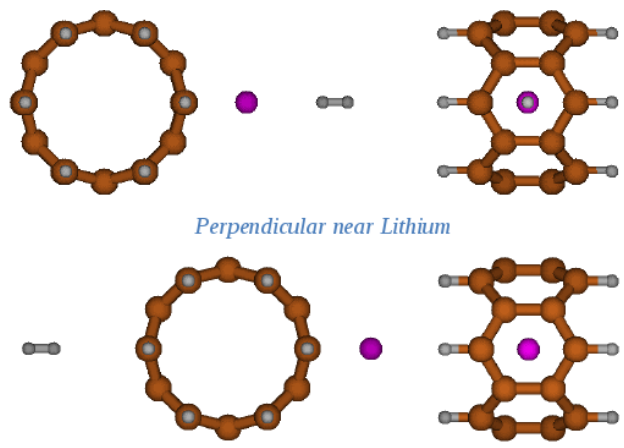

Perpendicular far from Lithium

Fig. 7. Perpendicular orientations of Hydrogen-Li-CNT interactions.

Interaction Potential of Hydrogen gas with Lithium-doped SWCNT (df-MP2: def2-SVP, Perpendiculi

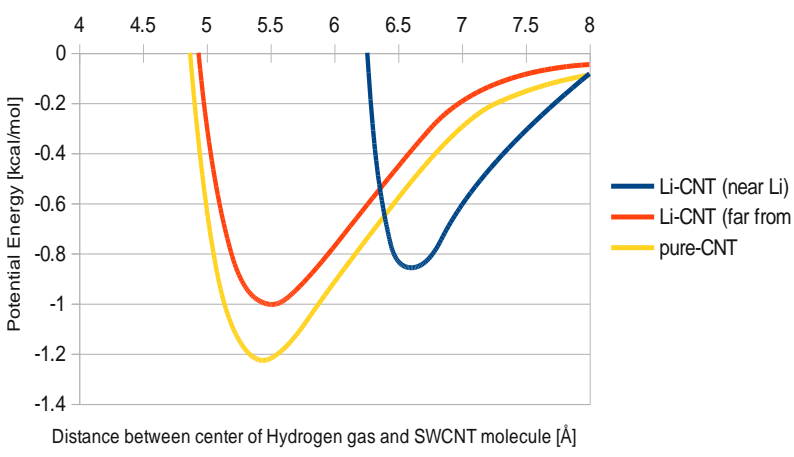

Fig. 8. Hydrogen physisorption energy on Lithium-doped CNT in perpendicular orientation.

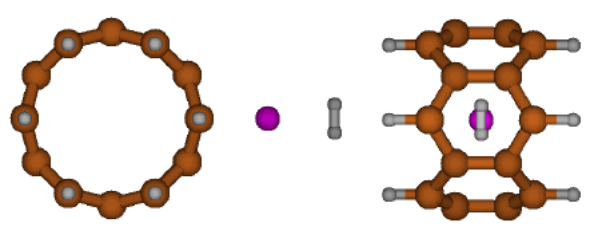

Parallel near Lithium
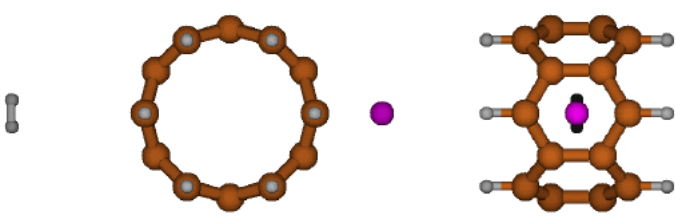

Parallel far from

Fig. 9. Parallel orientations of Hydrogen-Li-CNT interactions.
Interaction Potential of Hydrogen gas with Lithium-doped SWCNT (df-MP2: def2-SVP, Parallel)

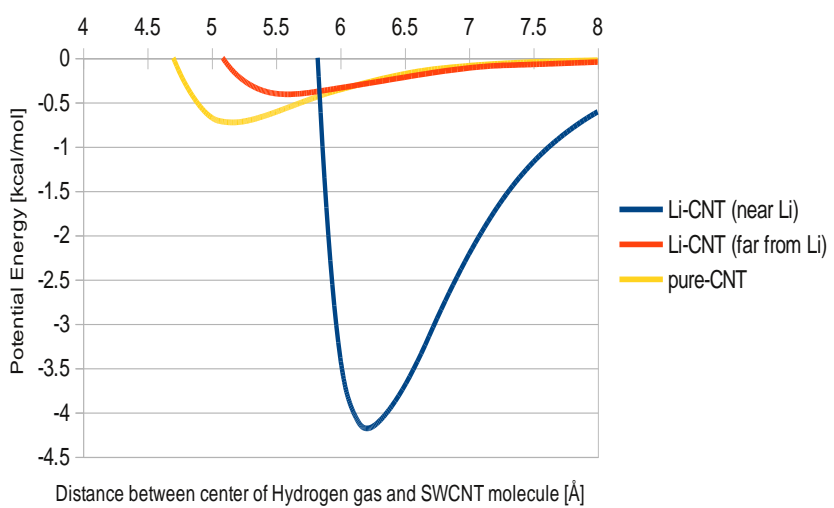

Fig. 10. Hydrogen physisorption energy on Lithium-doped CNT in parallel orientation.

\section{ACKNOWLEDGMENT}

We are grateful for kindness of our colleagues from LTT-RWTH Aachen for warmful discussions while one of our research group members visit their institute for two months. This research is supported by Research Grant of "PUPT-BOPTN-UI-2013" by Ministry of High Educations, Republic of Indonesia.

\section{REFERENCES}

[1] L. Simpson, "HSCoE final report 2010," Colorado: U.S. Department of Energy, 2010.

[2] M. Sankaran, B. Viswanathan, and S. Murthy, "Boron substituted carbon nanotubes-how appropriate are they for hydrogen storage?" International Journal of Hydrogen Energy, vol. 33, pp. 393-403, 2008.

[3] A. C. Dillon, K. M. Jones, T. A. Bekkedahl, C. H. Kiang, D. S. Bethune, and M. J. Heben, Nature, vol. 386, pp. 377-379, 2000.

[4] R. T. Yang, "Hydrogen storage by alkali-doped carbon nanotubes-revisited," Carbon, vol. 38, no. 4, pp. 623-626, 2000.

[5] J. H. Cho and C. R. Park, Catalysis Today, vol. 120, pp. 407-412, 2007.

[6] S. S. Han and H. M. Lee, Carbon, vol. 42, pp. 2169-2177, 2004.

[7] J. M. Turney, A. C. Simmonett, R. M. Parrish et al., WIREs: Computer Mol. Science.

[8] Z. Zhou, J. Zhao, Z. Chen, X. Gao, T. Yan, B. Wen, "Schleyer PvR," J. Phys. Chem. B., vol. 110, pp. 13363-13369, 2006.

[9] P. Chen, X. Wu, J. Lin, K. L. Tan, Science, vol. 285, pp. 91-93, 1999.

[10] R. T. Yang, Carbon, vol. 38, pp. 623-626, 2000.

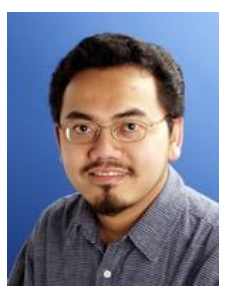

Ir. Nasruddin is a lecturer in the Department of Mechanical Engineering Faculty of Engineering University of Indonesia. He was born in Jakarta on April 11, 1972, he earned bachelor degree in mechanical engineering, University of Indonesia in 1995. He completed his master education of mechanical engineering in 1999 at KU Leuven, Belgium and doctoral education of mechanical engineering at RWTH Aachen, Germany in 2005.

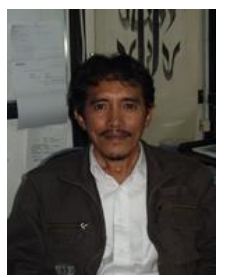

Engkos A. Kosasih is a lecturer in the Department of Mechanical Engineering Faculty of Engineering University of Indonesia. He was born in Tasikmalaya on June 6, 1959, he earned bachelor degree in Mechanical Engineering University of Indonesia in 1991. He completed his master education in mechanical engineering, Bandung Institute of Technology in 1997. 